Perspectiva Geográfica

ISSN 0123-3769 (Impreso) - 2500-8684 (En línea)

Vol. 21 No. 1 de 2016

Enero - Junio

pp. 127-150

\title{
Aproximación a la gestión del ordenamiento territorial en Colombia frente a los megaproyectos de infraestructura ${ }^{1}$
}

\author{
Approach to the Management of Land Use in Colombia Against \\ Large Infrastructure Projects
}

\section{Jahir A. Gutiérrez O. ${ }^{2}$ \\ Gleidy A. Urrego E. ${ }^{3}$ \\ Jefferson Carmona M. ${ }^{4}$}

Para citar este artículo utilice el nombre completo así:

Gutiérrez, J., Urrego, G. \& Carmona, J. (2016). Aproximación a la gestión del ordenamiento territorial en Colombia frente a los megaproyectos de infraestructura. Perspectiva Geográfica, 21(1), 127-150.

1 Este artículo es producto del proyecto de investigación "Evaluación Geoestratégica del Departamento del Chocó en el Marco de las Trampas del Subdesarrollo: El G-8 AID para el Departamento del Chocó", del Grupo de Investigación Observatorio Público, financiado por la Institución Universitaria Tecnológico de Antioquia.

2 Economista de la Universidad de Medellín, Colombia, Ph.D. en Administración Pública, Atlantic International University (AIU), Honolulú, USA. Magíster en Desarrollo, Universidad Pontificia Bolivariana, Medellín, Colombia. Estudios en Especialización de Sistemas de Información Geográfica, Universidad San Buenaventura (USB), Medellín, Colombia. Investigador, Facultad de Ciencias Administrativas y Económicas, Institución Universitaria Tecnológico de Antioquia. jagogutierrez@gmail.com

3 Magíster en Hábitat, Universidad Nacional de Colombia. Administradora Pública, Escuela Superior de Administración Pública, ESAP. Institución Universitaria Tecnológico de Antioquia. gleidy.urrego@gmail.com

4 Administrador Público, Escuela Superior de Administración Pública -ESAP-. Estudios en Políticas Públicas con Enfoque en Derechos Humanos, Ministerio del Interior y Justicia -ESAP-. Institución Universitaria Tecnológico de Antioquia. jefersoncm8@gmail.com 


\title{
Resumen
}

Con el objetivo de aproximarse a la gestión del ordenamiento territorial (OT) en Colombia frente a los megaproyectos de infraestructura, el artículo desarrolla una revisión bibliográfica y analiza su aplicabilidad, concluyendo que se requiere la concepción de la planeación del ordenamiento territorial nacional, y que un congruente ordenamiento territorial podría resolver los problemas territoriales a escala urbano-regional en materias y disposición del uso del suelo, necesarios en los megaproyectos de infraestructura. Se concluye, además, que el ordenamiento territorial debe alentar la formación de cartas de navegación espacial, geográfica y territorial a escala nacional, departamental y local para auspiciar los proyectos de infraestructura que impactan las disposiciones del uso, ocupación y apropiación del territorio, y que, de paso, estos puedan intervenir en la discusión sobre la finalidad y configuración del territorio desde el orden jurídico hasta lo sociocultural.

Palabras clave: megaproyectos de infraestructura, ordenamiento territorial, planificación territorial.

\begin{abstract}
This paper develops a bibliographic review and its applicability analysis considering an approach to the planning of Colombian land management, facing today's infrastructure megaprojects. This leads to the conclusion that a national territorial planning conception is required and that suitable planning could solve the territorial problems at the urban, regional and national scale, with sufficient and suitable land disposition, which are necessary for both social and economic developments. This includes agricultural, industrial, urban and reserve areas, improved by adequate infrastructure projects. Additionally, the territorial planning should encourage the spatial, geographic and territorial formation charts, at the national, departmental and local levels, in order to sponsor the infrastructure projects that impact the territorial use, occupation and appropriation dispositions. At the same time, it can intervene in the discussion over territorial use and configuration, from the legal aspects to the social and cultural spheres.
\end{abstract}

Keyword: infrastructure megaprojects, territorial planning, management planning. 


\section{Introducción}

El objetivo de este artículo de reflexión es aproximarse a la gestión del ordenamiento territorial en Colombia, que ha jugado un papel en el campo de la legislación, por lo menos, en lo atinente a la promulgación de leyes destinadas a materializarlo. No obstante, es un poco abrumador plantear que, a pesar de ello, es insuficiente la gestión del territorio en el país, y que ha sido complicado definir un plan nacional de ordenamiento territorial que genere pleno interés, en especial, frente a los megaproyectos de infraestructura; asimismo, se atienden como un hecho común las múltiples tensiones a las disposiciones de los usos del suelo y, de paso, no ha podido resolverse el tema vinculado a la disposición y ocupación del suelo que, de manera integral, debería darse al territorio nacional a la hora de programar proyectos de infraestructura.

La estructura jurídica provista ${ }^{5}$ para el campo del ordenamiento territo-

5 En cuanto a normatividad referida a ordenamiento y planificación territorial en Colombia se tiene: Ley 9 de enero 11 de 1989; Constitución Nacional de 1991, artículos 58 y 288; Ley 388 de julio 18 de 1997; Decreto 150 de 1999; Ley 507 de 1999; Decreto 932 de 2002; Decreto 1337 de 2002; Decreto 1788 de 2004; Decreto 3600 de 2007; Decreto 4065 de 2008; Ley 1454 de 2011; Decreto 3680 de 2011. rial no solo tiene dificultades para determinar las competencias en el ámbito local entre los municipios y las diferentes corporaciones ambientales, sino también para mediar como mecanismo para entender el tema desde el orden nacional. Temas circundantes con este, como infraestructura, mercado de tierras, producción y transformación productiva, se toman de manera aislada a la hora de complementar las directrices postuladas en el ordenamiento con las que se requieren puntualmente; es decir, prevalece una amplia desorientación en lo que implica el componente sustantivo de los aspectos que debe contener un plan de ordenamiento nacional y la manera como debe derivarse a escala departamental y municipal.

La política nacional de ordenamiento territorial, a cargo de lo que implica la disposición y usos del suelo (agricultura, reservas, asentamientos humanos, entre otros), es un tema de la realidad nacional que no ha podido materializarse; se habla de fundamentos y sustento legal, pero, en realidad, cada caso o situación que alerta sobre la manera como es aprobada, intervenida o transformada alguna parte del territorio indica que se tiene como mampara una inoperante legislación de ordenamiento, 
que más concuerda con otra forma de división política nacional que un trabajo que efectivamente señale los quehaceres sobre los que podrían atenderse los requerimientos territoriales a establecerse.

La concepción nominal a la que ha estado expuesto el territorio ha servido para que este sea considerado un bien que no amerita mayor detalle a la hora de analizarlo y evaluarlo. En cierta medida, la discusión del territorio se problematiza desde muchas vertientes. La geografía clásica lo plantea como una superficie conformada por agua, tierra y espacio aéreo, que desde las ciencias políticas y el derecho corresponde a un orden jurídico, como elemento en la concepción de Estado, determinado por fronteras y una jurisdicción. Por otra parte, desde la geografía crítica se propone el territorio como una construcción sociocultural, una trama de significados y relaciones políticas, sociales y culturales, y es visible en la medida que se logre distinguir lo material (asociado con la distancia-localización o receptáculo) y lo social, es decir, entre las cosas y sus acciones (Santos, 1999).

La discusión planteada por la economía con relación al papel constante registrado por el territorio ha servido para que sea considerado como componente institucional, más que como un bien de disposición, uso o valor, que en poco contribuye para elevar el carácter que de la mano de la institucional se subroga a este a la hora de servir para la extracción, la intervención o la transformación en distintos escenarios de trabajo, en los que necesariamente caben la infraestructura y los proyectos internacionales. Por tanto, deben converger en la planificación territorial la discusión y la problematización del enfoque del territorio, a partir de la norma constitucional, las leyes, los gobernantes, los actores políticos, socioculturales y económicos, bajo dos ejes: el orden jurídico y las posturas diferenciales frente a la apropiación y configuración del territorio.

En este sentido, la infraestructura civil e ingenieril en todos los escenarios, en especial la de mayor alcance, carece de un mapa de navegación claro para dar tránsito efectivo y real a los proyectos previstos en los programas internacionales del Banco Mundial en materia de construcción y edificación de los megaproyectos de infraestructura. La articulación de estos temas obedece a la pregun- 
ta: ¿es posible la gestión del ordenamiento territorial en Colombia frente a los megaproyectos de infraestructura? En esa medida, en el presente escrito los megaproyectos de infraestructura se inscriben en dos planos territoriales: el primero es la localización hecha a través de la cartografía, es solo un punto de referencia para ubicar las probabilidades de desarrollo de los proyectos en el país, y el segundo son las condicionantes ecológicas y socioculturales de la realidad de cada uno de los puntos localizables, particularmente, los de mayor calado, los cuales presentan dificultades que restringen su consecución rápida. Si, efectivamente, se contara con un plan de ordenamiento nacional en el país, no tendrían espacio las exaltaciones o las querellas presentadas a favor o en contra de la ejecución de diversas obras importantes para el país, que, para los no entendidos, dicho plan no puede utilizarse como mecanismo de solución pública estatal.

\section{Fundamentos de la interrela- ción entre el ordenamiento terri- torial nacional y los megaproyec- tos de infraestructura}

El ordenamiento territorial tiene como propósito evidenciar las condiciones y características del territorio frente a las perspectivas de la población en cuanto a la planeación; más que linderos jurídicos, trata de compatibilizar las intervenciones de los territorios con la capacidad territorial para absorberlos; a su vez, se convierte en un mecanismo para la disposición, gestión y usos del suelo, con los que debe mantenerse el equilibrio territorial. No obstante, el ordenamiento solo se entiende, en el caso colombiano, como la manera de permitir accesos al territorio sin mayores reparos, con la sola entrega de autorizaciones, licencias o permisos. Expone el Área Metropolitana del Valle de Aburrá:

La importancia de aplicar y desarrollar mecanismos de intervención en el suelo con el fin de vincularlos en obras públicas y proyectos estratégicos, radica en que el Estado debe extremar sus esfuerzos para ser eficiente en el uso de los recursos públicos y recuperar las plusvalías que él mismo genera (2010, p. 172).

El esperado marco jurídico general de ordenamiento territorial en Colombia es otra de las tareas incumplidas por los legisladores del país, 
que dejan la discusión sobre los territorios a cuenta del análisis de la disposición de la población frente al voto. Es cuestionable que el ordenamiento solo sea concebido como tutela de gestión en ciudades o lugares de mayor concentración poblacional, y que, para el caso de las áreas pequeñas o rurales termine siendo mas bien un elemento administrativo que no pasa a hacer parte de la trazabilidad que en materia de gestión territorial debería encumbrar la atención, precisamente, por lo que implica para ellas en cuanto a los usos de los recursos.

La compatibilidad entre los planes de desarrollo local con los planes de ordenamiento territorial de manera estructural es esquiva en la realidad nacional. La sola prevalencia de versiones contrarias en cada administración sobre lo que implica intervenir el territorio desdice precisamente del caudal de análisis que requiere tomar decisiones al amparo del ordenamiento. En Colombia, las administraciones municipales, en especial, toman el territorio con la misma atención que prestan a sus intereses políticos; solo contemplan el territorio desde el ordenamiento, como un espacio en el que puedan compatibilizar sus intereses de gobierno con los administrados en cada localidad. Analiza Mancero:

Si tomamos en consideración que el subsuelo es de propiedad del Estado, y que los particulares acceden al derecho de explotación del mismo a través de títulos habilitantes, la determinación de un derecho legal, válido e incontrovertible otorgado por el Estado, es fundamental cuando se busca adquirir derechos en el sector (2012, p. 25).

El recorrido un tanto empinado que deben cursar las obras de infraestructura para asentarse efectivamente en los lugares de oficio es solo una muestra de la desconexión entre las prerrogativas del ordenamiento territorial y las políticas encumbradas en los planes de desarro1lo. La sola réplica de la población, si bien es un derecho, escenifica el poco evidente trabajo permanente que debe guiar cualquier interés de perpetrar el territorio para la construcción, edificación e infraestructura, conforme a la prioridad en cada caso. En sí, el territorio pasa a reconocerse desde lo físico y geográfico a lo poblacional-territorial, vínculo que en contados casos es fraguado por los proyectos de infraestructura en general. 
Caracteriza Altschuler:

Es decir, que la proximidad del gobierno local respecto del territorio y del tamaño de la población no suponen por sí mismas una mayor interrelación entre el gobierno local y el sector privado o el tercer sector. Sino que muchos casos, por el contrario, en los municipios más pequeños, el nivel de articulación del gobierno con las organizaciones existentes en el territorio, dadas las carencias técnicas e institucionales a que antes hicimos referencia, son muy bajos o nulos. En los casos en que sí se da esta relación, generalmente, no se traduce en acciones orientadas al desarrollo local, sino que trata de prácticas tradicionales de clientelismo o el asistencialismo (2006, p. 6).

La prevalencia del ordenamiento, en tanto composición básica, esquema y plan en propiedad, contemplada para solventar el análisis conforme a la categoría municipal de nivel medio, bajo o alto, respectivamente, no termina por convencer eficazmente a las instancias que deben corroborar el espacio geográfico y territorial municipal con la planeación para el desarrollo departamental o local. Entre tanto, la mayoría de los mapas sobre ordenamiento terminan por enlistar, solo de manera puntual, las prioridades que una administración conciba para sí sobre lo que debe ser el ordenamiento en cada caso. Por tanto, el ordenamiento continúa bajo la lógica política local condicionante de los principios técnicos en sí. Argumenta Celedón:

Los municipios en desarrollo de la obligación de ordenamiento territorial, deben realizar la clasificación de suelos (urbano, rural, de expansión), definir los requerimientos y localización de las infraestructuras de transporte, vías urbanas, servicios públicos domiciliarios, el manejo de los residuos sólidos, la localización de las viviendas de interés social, los requerimientos y localización de parques, centros de producción, actividades terciarias, y zonas no urbanizables, entre otros asuntos. Las áreas de reserva, protección, ecosistemas municipales, y áreas de recuperación por desastres, son también de competencia municipal, las cuales deben atenderse a través de estos planes de ordenamiento (2006, p. 6). 
En tanto, el ordenamiento territorial es de competencia municipal y posibilita los megaproyectos de infraestructura en una lógica de desarrollo local que reduzca desequilibrios socioeconómicos y potencialice los determinantes ecológicos en el ordenamiento y planificación del territorio (artículo $10^{\circ}$, Ley 388 de 1997): áreas de conservación, áreas forestales, áreas protegidas, áreas cuencas, áreas de riesgo, clasificación del suelo, áreas de protección agrícola y áreas patrimoniales. La destinación de áreas para diversas disposiciones y usos, a la par con la indicación de los espacios en los cuales albergar grandes proyectos, hacen parte de las competencias municipales. El propósito del esquema de planeación territorial frente a megaproyectos de infraestructura (ver Tabla 1) habilita las condiciones de intervención territorial conforme a las características geográficas y patrones de ocupación del suelo.

La consecución de un plan nacional de ordenamiento territorial podría convertirse en la primera base para entender los compromisos que en materia espacial y geográfica deberían acoger la mayoría de proyectos de infraestructura. En esta materia, las escalas de ordenamiento territorial departamental y municipal se cobijan en la política nacional de ordenamiento territorial, donde el departamento consolida a escala regional (más allá de los límites político-administrativos) todos los determinantes ecológicos del artículo 10, Ley 388 de 1997 de la escala municipal y el tipo e impacto social, ambiental y poblacional de los megaproyectos de infraestructura que incide en ellos. A su vez, orientar toda la infraestructura física-social que despliega los megaproyectos, de manera que sean aprovechables las ventajas comparativas.

Por su parte, el municipio valora y somete todo megaproyecto de infraestructura realizable en su territorio a los principios de la Ley 388 de 1997 en su artículo $2^{\circ}: 1$ ) La función social y ecológica de la propiedad. 2) La prevalencia del interés general sobre el particular. 3) La distribución equitativa de las cargas y los beneficios. Esto evidencia la tensión que emerge entre los diferentes grupos poblacionales, los tipos de megaproyectos, los sistemas determinantes en la planificación del territorio y las escalas de ordenamiento territorial basadas en una división demográfica. 
Tabla 1. Escalas del ordenamiento territorial frente a los megaproyectos de infraestructura

\begin{tabular}{|c|c|c|c|}
\hline Escalas OT & $\begin{array}{c}\text { Sistemas } \\
\text { determinantes en } \\
\text { la planificación del } \\
\text { territorio }\end{array}$ & $\begin{array}{l}\text { Megaproyectos de } \\
\text { infraestructura }\end{array}$ & $\begin{array}{l}\text { Tensiones territoriales en } \\
\text { torno a los megaproyectos } \\
\text { de infraestructura (grupo } \\
\text { poblaciones, especialmente } \\
\text { Comunidades Indígenas y } \\
\text { Afro descendientes) }\end{array}$ \\
\hline \multirow{5}{*}{$\begin{array}{l}\text { 1. Plan de } \\
\text { Ordenamiento } \\
\text { Territorial POT } \\
\text { más de } 100.000 \\
\text { hab. 2. Plan Básico } \\
\text { de Ordenamiento } \\
\text { Territorial entre } \\
\text { 30.000 y } 100.000 \\
\text { hab. 3. Esquema } \\
\text { de Ordenamiento } \\
\text { Territorial menos } \\
\text { 30.000 hab. }\end{array}$} & \multirow{5}{*}{$\begin{array}{c}\text { Equilibrio entre el } \\
\text { sistema natural y } \\
\text { sistema artificial que } \\
\text { comprende: } \\
\text { 1. Sistema natural: } \\
\text { Estructura ecológica: } \\
\text { corredor ambiental } \\
\text { equilibrio entre } \\
\text { factores bióticos y } \\
\text { abióticos. y comprende } \\
\text { los determinantes } \\
\text { ecológicos del artículo } \\
\text { 10, Ley } 388 \text { de } 1997 . \\
\text { 2. Servicios ambientales: } \\
\text { son los beneficios } \\
\text { proporcionados de } \\
\text { forma natural y se } \\
\text { generan de la estructura } \\
\text { ecológica. } \\
\text { 3. Sostenibilidad: } \\
\text { Sistema artificial: lo } \\
\text { urbano, intervenciones } \\
\text { en infraestructura }\end{array}$} & \multirow{3}{*}{$\begin{array}{l}\text { Energía (Embalses -hidro- } \\
\text { energéticos, transporte } \\
\text { (vías), suministro de agua } \\
\text { y saneamiento, servicios } \\
\text { urbanos, telecomunicaciones, } \\
\text { petróleo, gas, minería. De } \\
\text { acuerdo al Banco Mundial es } \\
\text { buscar mejorar la prestación } \\
\text { de servicios de infraestructura, } \\
\text { a través de reformas, creación } \\
\text { de inversión en activos físicos } \\
\text { para el desarrollo de los } \\
\text { países. }\end{array}$} & $\begin{array}{c}\text { Cambios en los usos del suelo } \\
\text { urbano-rural }\end{array}$ \\
\hline & & & $\begin{array}{c}\text { Propiedad, uso y gestión de } \\
\text { la tierra }\end{array}$ \\
\hline & & & $\begin{array}{c}\text { Conflictos socioambientales } \\
\text { por el uso y disposición del } \\
\text { agua, suelos de conservación } \\
\text { y reservas campesinas para la } \\
\text { agricultura }\end{array}$ \\
\hline & & \multirow{2}{*}{$\begin{array}{l}\text { Los tipos de infraestructura se } \\
\text { enmarcan en el cumplimiento } \\
\text { de los objetivos del milenio, } \\
\text { a su vez, la alianza de los } \\
\text { sectores público y privado } \\
\text { en materia de asesorías, } \\
\text { políticas públicas y fortalecer } \\
\text { la coordinación de donantes } \\
\text { que promuevan infraestructura } \\
\text { (Banco Mundial, 2004) }^{6}\end{array}$} & $\begin{array}{l}\text { Disputa por recurso minero } \\
\text {-energéticos }\end{array}$ \\
\hline & & & $\begin{array}{l}\text { Conflicto, violencias, } \\
\text { desplazamientos }\end{array}$ \\
\hline
\end{tabular}

Fuente: Elaboración propia.

6 "Acceso a obras de infraestructura, calidad y necesidades de financiamiento: Aunque existe un consenso generalizado respecto de la importancia de las obras de infraestructura, persiste la poca claridad en relación con el acceso, la calidad y las necesidades de financiamiento para estos servicios. Por ejemplo, en las zonas rurales de países de ingresos bajos, solo el $20 \%$ de la población tiene acceso a electricidad. Este desafío se ve agravado por la mala calidad de los servicios disponibles. Una comparación entre los países de ingresos bajos y los países de la OCDE arroja el doble de cortes de energía, cuatro veces más cortes de agua, diez veces más desperfectos en las líneas telefónicas y sólo el $29 \%$ de caminos pavimentados en los primeros en comparación con más del 80 \% en los segundos. Un estudio del 2004 realizado en siete países de América Latina sugiere que la mala calidad de la infraestructura pública es responsable de la efectividad de sólo el $74 \%$ respecto de los países industrializados y que el costo a largo plazo relacionado es equivalente a cerca del $40 \%$ del ingreso per cápita.

Solo con grandes inversiones se podrá conquistar el desafío de aumentar el acceso a buenos servicios de infraestructura. Las estimaciones actuales indican que las necesidades de financiamiento suman alrededor del $7 \%$ del PIB en todos los países en desarrollo, tanto para gastos en nuevas inversiones como para mantenimiento. Las necesidades de financiamiento en los países de bajos ingresos pueden llegar al $9 \%$ del PIB. Una comparación entre la inversión real en el pasado y las tasas de gastos para inversiones y mantenimiento (en promedio cercanas al 3,5 \% del PIB en todos los países en desarrollo) con las exigencias proyectadas, apunta claramente hacia la necesidad de duplicar el financiamiento real para obras de infraestructura. Por ende, se necesitan muchos más recursos para aumentar el acceso a buenos servicios de infraestructura" (Banco Mundial, 2004). 
Ello se traduce en: 1) Grandes proyectos de interés nacional y departamental que condiciona la planificación local. 2) Relación del suelo/ subsuelo, el primero de propiedad privada y el segundo propiedad estatal, ejemplo: minería frente los usos del suelo. 3) Consolidación de los determinantes ecológicos imperantes en todo el megaproyecto más allá de los suelos de proyección. Establece Revéiz:

La Política Nacional de Ordenamiento Territorial (PNOT) debe repensar instrumentos simplificados y articulados entre los diferentes órdenes territoriales $\mathrm{y}$ el sector privado para fortalecer la democracia y la sociedad civil, dando solución a varios tipos de conflicto, reasignar funciones del Estado y articular en el territorio las competencias del Estado Providencia, del Estado Estratega, del Estado Protector y del Estado Regulador de Riesgos. Es prioritaria la creación del Ministerio del Ordenamiento Territorial para anticipar la aparición y profundización de los conflictos territoriales, para proponer estrategias de polos de competitividad dentro del marco de las APP y eliminar la paranoia normati- va intergubernamental. En esta parte se presentan algunos de los conflictos territoriales emblemáticos que surgieron por la "inflación exponencial" de concesiones petroleras y mineras y el mal manejo de los derechos especiales de uso y ocupación del territorio nacional. Debe crearse un Banco de Conflictos Territoriales (BCT) que ilustre las experiencias exitosas y fallidas de manejo de conflictos en el territorio (2013, p. 17).

Los proyectos de infraestructura están a la intemperie en cuanto a la posibilidad de emprender obras y culminarlas con éxito. Más allá de las concesiones, legalidades, pólizas y requisitos, el suelo sobre el que tratan de desempeñar sus labores aún no goza de argumentos técnicos sobresalientes sobre los cuales advertir o, definitivamente, no poner en marcha ningún tipo de iniciativa. Es sumamente criticable que en el país se fomente el desarrollo de grandes proyectos cuando de manera paralela se tienen dificultades para establecer los argumentos sobre los cuales extender dichas obras, escollo que no han podido superar los proyectos, generalmente, y que se convierte en un lastre para su efectiva gestión. 


\section{Metodología}

El método utilizado en este estudio es descriptivo, a partir del objetivo de aproximarse a la gestión del ordenamiento territorial en Colombia frente a los megaproyectos de infraestructura, desde la articulación entre el ordenamiento territorial y la programación de proyectos de infraestructura. El diseño del estudio se basa en dos fases: i) etapas de revisión bibliográfica, informes y normatividad sobre ordenamiento territorial y megaproyectos de infraestructura, y ii) análisis de su aplicabilidad. Se basa en dos categorías centrales: territorio y megaproyectos, con dos variables: ordenamiento jurídico del territorio e infraestructura.

\section{Resultados}

\subsection{Aproximación a la evaluación de la capacidad territorial para absorber los impactos de los me- gaproyectos de infraestructura}

La incorporación de proyectos en distintas áreas y zonas implica una evaluación amplia en cuanto a lo que atañe a la capacidad de absorción territorial de ellos. No bastan las constantes réplicas dirigidas a promulgar la trascendencia de las obras; el proceso de internalización de ellas es tarea que corre por cuenta de todos los actores involucrados en ellas. Incluso, por fuera de los términos entre el inicio y la finalización de la obra, la gerencia de proyectos debe dedicar parte de su trabajo a continuar divulgando el alcance, el logro y la transversalidad de las obras construidas, temario que aún no hace parte de los recursos administrativos y financieros en los que se entrelazan los beneficios y costos establecidos en los procesos de contratación. Explica Celedón:

La Ecoeficiencia, como proceso y metodología ambiental y productiva, gracias a su aplicación exitosa en las actividades económicas, hace su ingreso firme en el terreno público, en especial, en los sectores que mayor impacto generan en el bienestar de la población: el sector de las infraestructuras urbanas, entendidas éstas como aquellas estructuras físicas, que le brindan soporte a las actividades y funcionamiento indispensables para la vida urbana $(2009$, p. 5).

El contexto en el que se disertan los temas inherentes a los megaproyectos de infraestructura está más del 
lado de los cuestionamientos y los procesos legales que de la lógicaargumentación destinada a congeniar las perspectivas embarcadas en los proyectos. En dicho sentido, la gestión territorial de los proyectos no hace parte de la gerencia técnica de ellos, por cuanto se asume que en estas circunstancias los amparos jurídicos y legales contienen los elementos requeridos para que las obras tengan respaldo. No obstante, los proyectos solo tienen respaldo a la luz de los requisitos que han de cumplir, que como la mayoría de precondiciones no advierten de los altibajos y problemas que puedan presentarse en obra. Establece el Banco Interamericano de Desarrollo:

Por sus características de multiplicador en todo el aparato productivo, en términos de encadenamientos en otros sectores productivos, la infraestructura tiene un efecto directo sobre el crecimiento, la productividad y la integración. Para el caso colombiano, con una geografía tan difícil, una mejor infraestructura permite reducir las barreras naturales para la comunicación, el transporte y el intercambio con el fin de incrementar el aparato productivo en los mercados internacionales (2000, p. 193).
Es tarea de los departamentos y municipios disponer de áreas geográficas y territoriales saneadas de toda condición. La animadversión presentada con cada ejecución o puesta en marcha de obras podría superar el perfil mediático si se extendiera el alcance de las obras programadas o previstas por cuenta de las autoridades componentes. El estudio sobre los proyectos solo obedece al componente ingenieril y técnico, y muy poco a los lineamientos y trazabilidad que por cuenta de estos deben integrarse a las exaltaciones presentadas por la población; esto último implica un costo de transacción y de externalidades que, al parecer, no está en las cuentas de nadie, por cuanto ni siquiera las toman en cuenta. Ejemplariza el Consorcio GTZ/ Fundeco/IE:

La información recabada para cada uno de los países permitió realizar un análisis FODA sobre la relación entre megaproyectos y biodiversidad en la región. Entre las principales debilidades encontramos que todavía no existe un buen nivel de interacción entre las dependencias de las instituciones públicas que manejan los temas de biodiversidad con las que manejan 
los temas del desarrollo; no hay un concepto unificado sobre la función específica de un EIA, como herramienta que integra la biodiversidad en los proyectos, aunque la gestión de la biodiversidad en megaproyectos ha tenido mejor atención para algunos sectores más grandes, como los energéticos (petróleo y gas), mineros o de transportes. Los países de la subregión, en general, todavía no poseen sistemas en funcionamiento de valoración económica de la biodiversidad, situación que hace que los impactos de los megaproyectos no pueden ser valorados de manera adecuada (2002, p. 5).

El perfil dado a los planes de desarrollo departamental y municipal continúa en la margen de las expectativas y sueños establecidos por quien asume el poder, dejando el territorio en el espacio de repositorio sobre el cual pretenden ahincarse dichas perspectivas. A pesar de la alusión e ilustración mimetizada en representaciones espaciales y geográficas, los territorios, en particular los municipios, tienen por fuera los patrones de integración que al alcance de ellos generan los proyectos establecidos desde el orden central, nacional y regional. En general, los planes de desarrollo no recogen las cargas territoriales creadas por los proyectos de infraestructura, y menos los impactos que podría traer a escala local y regional. Arguyen Silva y Gonzáles:

Los desafíos sociales, políticos y económicos que hoy todavía son vigentes en los países en vías de desarrollo requieren de procesos complejos e innovadores que permitan transformar los sistemas de administraciones locales y regionales, en gobiernos que aseguren la participación ciudadana estructurada, que asignen recursos con visión de largo plazo, que permitan el ordenamiento del territorio $\mathrm{y}$ el uso eficiente de los recursos naturales disponibles. Asimismo, es primordial que generen un desarrollo económico territorialmente más equilibrado, y la toma de decisiones que conlleven mejorar las condiciones para el desarrollo humano y social de la población (2010, p. 90).

La ejecución de los megaproyectos no pasa por el diálogo, la evaluación y la prescripción de las autoridades y la población municipal 
en donde tienen asiento, impacto o trascendencia; a cuentagotas son desarrolladas algunas actividades informativas y de interacción cuando las obras se encuentran en marcha, y detrás de ellas quedan dificultades que deben pasar a revisión de los hacedores de los proyectos, dificultades que emergen a medida que la obra provoca animadversiones o pretensiones de todo tipo. Es poco factible que aflore el éxito de un proyecto de obra o infraestructura cuando la gerencia de proyectos es poco evidente o visible en general. Expone Lozano:

Cuando a un inversionista o a un pequeño productor se le presenta un precio de oferta descubre que cuenta con muy pocos elementos técnicos o económicos para argumentar una contrapropuesta; aunque generalmente se le ha presentado un valor circunstancialmente establecido. Para mejorar el proceso de toma de decisiones acerca de las potenciales inversiones agropecuarias y para el desarrollo de proyectos de infraestructura que impulsen el desarrollo regional, es indispensable que el inversionista conozca de antemano cuáles son las variables que inciden en el comportamiento del mer- cado de las tierras y, segundo, cuál es el peso que ellas tienen en la determinación del precio final (2007, p. 2).

En manos de las autoridades y de la población de donde tienen lugar los proyectos de infraestructura está la oportunidad de establecer los puentes de trabajo entre el territorio y los interesados en poner en marcha los proyectos. El sobresalto que acontece entre la población y los ejecutores del proyecto es el espacio que propicia el no trabajo permanente de los diferentes entes que deben responder en cada paso con lo que acontezca en atención a los episodios presentados en los proyectos. Se debe articular la ejecución de los proyectos con los diferentes actores que inciden en la consecución, obra y terminación de ellos, y que deben generar elementos de permanente estudio, gestión y análisis. Ejemplariza Páez:

La mayoría de las corporaciones autónomas regionales no han realizado una ordenación forestal que les permita saber: - ¿Qué sucede con sus ecosistemas boscosos?; ¿Cómo están siendo transformados?; ¿Qué se ha perdido y se está perdiendo en estos ecosistemas?; ¿Qué 
procesos ecológicos sociales y ambientales, ocurren cuando se presentan transformaciones ocasionadas por actividades antrópicas o por factores naturales?; ¿Cuál es su capacidad de ejecución en la gestión del riesgo ante la transformación negativa de estos ecosistemas?; ¿Cuál es la tasa de pérdida anual de bienes y servicios ambientales con estas transformaciones? (2010, p. 91).

La evaluación profunda sobre lo que implica acceder o permitir el ingreso de diferentes actores para la generación de proyectos de carácter territorial debe superar el proceso propio cohonestado en consultas o referendos. El estudio constante de lo que implica para un municipio establecer o admitir la presencia de diferentes proyectos de infraestructura en diversas zonas obliga, necesariamente, a establecer criterios de trabajo sobre los cuales atender los diversos contextos sociales, económicos y políticos sobre los que el proyecto se desenvuelve, lo cual debe correr por cuenta, precisamente, de las autoridades territoriales, que deben estar al tanto de los impactos que desde diferentes ámbitos genera la consolidación de megaobras en los diferentes territorios. Analiza Gonzáles:

Las comunidades indígenas y afro-descendientes se preguntan por las obligaciones y responsabilidades que tienen las empresas o proyectos de inversión, con la sociedad, las comunidades y los derechos humanos de los grupos que se relacionan directamente con alguno de los componentes de los negocios que se piensan emprender o se están desarrollando. Las respuestas a estas preguntas han dado lugar a muchos debates e incluso es un tema de discusión actual de las Naciones Unidas y en su Comisión de Derechos Económicos y Sociales. Preguntas para reflexionar: ¿Cuáles son las obligaciones del Estado y cuáles de las empresas?; ¿Ante quién tienen que responder las empresas si cometen un abuso o son cómplices de violación de algún derecho?; ¿Qué responsabilidad corresponde a los planes de desarrollo e inversión del Estado cuando afectan territorios y planes de vida de comunidades indígenas $\mathrm{o}$ afro-descendientes?; ¿Qué derechos pueden reclamar las comunidades étnicas frente a programas de inversión 
que afectan sus condiciones de existencia? (2011, p. 109).

Es inconcebible que entre diferentes autoridades y entidades dedicadas a la gestión de proyectos desde los frentes ambientales, ecológicos, políticos y de planeación, prevalezcan, además de desavenencias, diversas versiones frente a lo que implica autorizar, permitir o emprender proyectos de infraestructura. Es esta situación discordante la que indica el momento de desatención o pesadumbre que deben sobrellevar los diferentes actores interesados en llevar a cabo los proyectos de infraestructura, para los que las respuestas o salvedades parecen saltar más por las desavenencias de los estudios que por criterios juiciosos y profundos sobre los que pueda atenderse la versión de otras fuentes, con el propósito de poner a disposición y en detalle lo que implica emprender el proyecto.

\subsection{Disposición y usos del suelo de los territorios para la cons- trucción de megaproyectos de in- fraestructura}

La disposición de suelos para los frentes de proyectos de construcción e infraestructura de grandes obras no hace parte de la oferta que se tiene por el momento para ellos. En esencia, la mayoría de los programas de proyectos no tienen, básicamente, el reconocimiento del terreno sobre el cual se intervendrá. Es una situación preocupante, si se considera que los proyectos tienen unos tiempos en las distintas etapas que pueden alargarse cuando la dotación básica, como el propio suelo, ni siquiera está dispuesto o negociado de manera efectiva para llevarlos a cabo. Es controversial que se ofrezcan distintos proyectos sin contar con la oferta de suelos al día. Considera Sassen:

Una tendencia principal evidente en todo el mundo es la formación de escalas urbanas crecientemente grandes, las cuales en cierto momento pueden ser descritas como mega-regiones. A menudo, ellas son simplemente vistas como más de lo mismo -más personas, más paisajes urbanos interminables. En su aspecto más elemental, la mega-región resulta del crecimiento de la población en un contexto geográfico donde ciudades y áreas metropolitanas se mezclan entre sí. Y esto, en efecto, conduce a infraestructuras interregionales, notable- 
mente transporte y electricidad, y varias formas de planificación y coordinación regional, como puede ser observado hoy. Pero, ¿son estas condiciones, que implican una versión expandida de las economías de urbanización, todo lo que hay que considerar? (2007, p. 11).

Al no disponer de la oferta de suelos necesarios para el tránsito de los megaproyectos de infraestructura, deben alentarse las propuestas dirigidas a la programación de proyectos en los diferentes campos de trabajo, y que esta matriz, igualmente, tenga espacio de revisión por diferentes actores interesados, más que en los detalles ingenieriles, en las consecuencias presentadas en los suelos expuestos a la intervención directa o a los impactos indirectos. El suelo contemplado en el área de intervención del proyecto, al igual que los interconectados con él, implica para todos ponderar los alcances y propuestas que de manera directa atraen la ejecución del proyecto o, incluso, su parálisis. Analiza Maldonado:

La Ley 388 de 1997, o Ley de desarrollo territorial, establece una serie de instrumentos con los que pretende cumplir objetivos diversos y complejos. Se puede afirmar que uno de sus principales méritos es el de plantear una articulación más explícita entre plan e instrumentos de gestión del suelo que la que existía en la Ley $9^{a}$ de 1989. Mas aún, podría decirse que más que una simple ley de planeación o de ordenamiento físico o territorial es una ley del suelo (2006, p. 1).

El suelo dispuesto para la ejecución de obras e infraestructura del país está por fuera del alcance del Estado y de los concesionarios interesados en desarrollar los proyectos. En cada tramo que logran avanzar, los proyectos deben atender dificultades propias de la adquisición de predios y, de paso, el licenciamiento para que estos cambien la forma de tenencia a la que respondían, para que terminen adheridos a los proyectos relacionados. En Colombia, los suelos para la infraestructura es un tema que logra en la actualidad atención, por cuenta de los problemas que en cuanto a indemnización, pagos y valorización de predios continúan debilitando los recursos ofrecidos para el trámite de la obra, pero no para el pago de suelos. Argumenta Jaramillo: 
Otro grupo importante de instrumentos de gestión del suelo y de imposición de la propiedad inmobiliaria no tiene como referente el precio total del inmueble o el precio total del suelo, sino sus incrementos. El aumento del precio del suelo, que normalmente se manifiesta también como aumento del precio del inmueble construido, se le conoce con la palabra de origen francés, plusvalía, que tiene aquí el sentido del precio de un bien, el término que tiene además la connotación de que este aumento, es algo inmerecido (2009, p. 336).

La oferta de suelos para proyectos relevantes en el país no hace parte del análisis que de manera clara debe ser atendido por el Estado antes de emprender cualquier proceso de concesión, licitación o proposición de un proyecto. El denominado interés público nacional aún no atiende las condiciones mínimas a las que debe apuntar la disposición de suelos para la elaboración de los proyectos; en contraste, los altos costos para adquirirlos, derivados de las plusvalías generadas por el interés que crean las obras o por la puesta en marcha de ellas, es el elemento en cuestión que se analiza a la hora de disponer de los suelos necesarios para cimentar los proyectos. Contextualiza Herrera:

Es justo mencionar que estas recomendaciones de políticas deben estar acompañadas de procesos de participación pública que tomen en cuenta a las comunidades que se ven afectadas con la puesta en marcha de proyectos tendientes a mejorar la situación de la tenencia de la tierra. Las discusiones sobre políticas de tierra no se pueden hacer sin considerar la realidad presente tanto en el campo como en las ciudades. Para lograrlo es necesario organizar programas de consulta a la sociedad civil. Esto también está relacionado con el grado de voluntad política y capacidad financiera que tienen los distintos entes involucrados en implementar la infraestructura necesaria, especialmente la inversión para modernizar y descentralizar servicios de registro y catastro. En la mayoría de los casos, el financiamiento de los gobiernos deberá estar acompañado de contribuciones externas, lo cual podrá originar preocupación entre los diseñadores de políticas públicas, pues no siempre se puede contar con 
los montos requeridos por parte de la banca multilateral o donantes bilaterales para satisfacer las expectativas nacionales. Será por lo tanto necesario acudir a mecanismos innovadores de financiamiento compartido entre organismos del sector público e instituciones del sector privado que se vean favorecidas con la presencia de sistemas de propiedad modernizados (2006, p. 10-11).

La economía del suelo para la disposición y uso de diferentes actividades productivas, que implican la transformación del territorio a escala de los megaproyectos de infraestructura, no hace parte del andamiaje de análisis presupuestales y administrativos en los que deben involucrarse las autoridades, los municipios y las empresas interesadas en llevar a cabo proyectos. En realidad, la poca información que se tiene frente a este rubro de inversión en proyectos, precisamente, es el principal obstáculo que deben sortear todos los sectores, aunque la posición individual y marginal es la que garantiza la manera como cada uno decide atender dicho bache: el Estado negociando los predios, la comunidad expo- niendo sus querellas y las empresas a portas de demandas.

La valoración económica del suelo hace parte de las tareas pendientes de la administración departamental y municipal, particularmente rural, del país. En esta valoración han sido articulados algunos impuestos, como catastro o predial, que están más al tanto de las construcciones y edificaciones de carácter familiar o comercial, que de una amplia determinación de lo que implica asentar impuestos desde las características y condiciones del suelo. La clasificación del suelo no hace parte de los elementos revisados por los definidores de impuestos, de allí que, cuando comienzan a adquirirse los predios alrededor de los proyectos, no se conoce ciertamente el valor de ellos. Establecen Sciara y otros:

El Banco Mundial, en su informe sobre Infraestructura de 1994, se centra en la infraestructura económica y la define como aquella que abarca lo siguiente: servicios públicos (es decir, energía eléctrica, telecomunicaciones, abastecimiento de agua por tubería, saneamiento $\mathrm{y}$ alcantarillado, recogida $\mathrm{y}$ eliminación de residuos sólidos 
y suministro de gas por tubería); obras públicas (como carreteras y obras importantes de presas $\mathrm{y}$ canales de riego y drenaje) $\mathrm{y}$ otros sectores de transporte (como ferrocarriles urbanos e interurbanos, transporte urbano, puertos y vías navegables y aeropuertos). También se la puede definir en función de la propiedad de los activos, identificando la infraestructura con el stock de capital físico en manos del Estado. En muchos estudios econométricos se adopta esta definición, para evitar dificultades en la medición del capital de infraestructura en manos del sector privado; en el caso argentino, sería una definición muy limitada, ya que existe una amplia gama de servicios públicos privatizados (2004, p. 6).

En cada tipo de megaproyecto de infraestructura acontece un fenómeno similar: disputas sobre la disposición y los usos del suelo, altos costos de los predios adquiridos $\mathrm{y}$ desavenencias entre diferentes autoridades, que, de manera mediática, avalan o reprochan cualquier situación presentada. El problema de la generación de proyectos de interés nacional está sustentada en la vulnerabilidad que cargan por cuenta de los improvisados elementos y mecanismos de gestión que, a la par con los propios de la infraestructura, deben evidenciarse en cuanto a la gestión administrativa y la gerencia de los proyectos en el frente del suelo y los territorios dispuestos para ello, en donde la labor supera el marco ingenieril. Expresa Carrillo:

La causa fundamental de la incomodidad de minorías y la preocupación del Gobierno es la ausencia de reglas de juego en implementación de la consulta previa. Esta es un mecanismo de enriquecimiento cultural, cuyo gran desafío es encontrar el equilibrio entre un aspecto económico y los aspectos propios de las comunidades. La consulta previa y el proceso no son ni serán un obstáculo para el desarrollo (2013, p. 17).

Es primordial que las empresas de construcción, infraestructura y megaproyectos destaquen la administración y gestión de suelos antes, durante y después del desarrollo de los grandes proyectos. En esencia, es de destacar que cada éxito obtenido en este frente puede servir de elemento de trabajo para los diferentes actores, en tanto contempla 
la labor que de manera directa deben acometer las compañías para hacer de las grandes obras un marco de gestión para cualquier otro tipo de intervención que se apreste para un mismo tratamiento o complementario a este. El conocimiento en materia de gestión de suelos y proyectos es una tarea que no ha sido cumplida por las grandes empresas de ingeniería del país.

\section{Conclusiones}

Es necesario problematizar la gestión del ordenamiento territorial en Colombia frente a los megaproyectos de infraestructura, lo que, indudablemente, abarca la normativa en materia de planificación territorial, para valorar los impactos de las intervenciones a escala urbano-regional y no a simple escala municipal. A su vez, se requiere dimensionar los megaproyectos de infraestructura a la luz de los determinantes ecológicos estipulados en el artículo $10^{\circ}$ de la Ley 388 de 1997, y no solo de las disposiciones del uso del suelo.

El ordenamiento territorial debe alentar la formación de cartas de navegación espacial, geográfica y territorial a escala nacional, departamental y local, para auspiciar los proyectos de infraestructura que impactan las disposiciones del uso, ocupación y apropiación del territorio, $y$, de paso, para que estas cartas puedan intervenir en la discusión sobre la finalidad y configuración del territorio desde el orden jurídico hasta lo sociocultural. El acusado señalamiento en el tema corresponde a que gran parte de lo que atenta contra la buena gestión de los proyectos corre por cuenta de la manera como las empresas, o el propio Estado, atienden las gestiones y los riesgos financieros y administrativos embarcados en los megaproyectos en materia de disposición y usos del suelo.

Los concesionarios de infraestructura y megaproyectos deben profundizar en materia de gestión de proyectos, en particular, en cuanto a suelos, población (afectación de grupos indígenas, afrodescendientes y demás) y territorio (sistemas naturales); en cada caso, más allá de la cuestión del proyecto, lo que inquiete a la población debe ser lo que aliente la gestión del proyecto. La combinación entre el gerente social y el gerente técnico del proyecto es crucial para que se tenga éxito en el tiempo. La definición de un marco propio de gestión de las empresas podría resolver más pro- 
blemas que los puntuales, que en la mayoría de los casos es lo que se cuestiona con cada proyecto en mención.

El Estado nacional debe alentar y disponer recursos para que las autoridades locales y regionales, además de las corporaciones encargadas de administrar o gestionar los impactos territoriales, desarrollen mecanismos de gestión tras los que puedan evaluar en el tiempo los alcances, elementos y prerrogativas que deberían acompañar los proyectos en sus diferentes etapas. La tarea cumplida hasta ahora por las autoridades locales y regionales y las corporaciones continúa siendo requisitoria, que en cualquier caso cualquiera podría cumplir; el problema radica en que no se conoce una evaluación plena de la manera como la comunidad atiende, evalúa y reciben los impactos de los megaproyectos por encima de las consultas previas.

\section{Referencias}

Altschuler, B. (2006). Municipios y desarrollo local. Un balance necesario. Buenos Aires: Espacios.

Área Metropolitana del Valle del Aburra (2010). Mecanismos de Intervención del Suelo (171204). En: Instrumentos de Gestión Urbana (pp. 1-247). Subdirección de Planificación Integral.

Banco Interamericano de Desarrollo (2000): Un nuevo impulso para la integración de la infraestructura regional en América del Sur. Recuperado de http//www.iadb.org/intal/ publicaciones/infraestructura/.

Banco Mundial. (2004). Reseña sobre la infraestructura. Recuperado en: http://www. bancomundial.org/temas/resenas/infraestructura.htm

Carrillo F. F. (2013). Vueltas con la consulta previa. El colombiano, Opinión, jueves 21 de febrero, p. 17.

Celedón, P. C. (2009). Ecoeficiencia e infraestructuras urbanas en Colombia. Comisión Económica para América Latina y el Caribe (CEPAL).

Consorcio GTZ/ Fundeco/IE (2002). Estrategia Regional de Biodiversidad para los países del Trópico Andino. Impacto de Megaproyectos de Infraestructura. Banco Interamericano de Desarrollo. 
González P. C. (2011). Renta minera, petróleo y comunidades. Bogotá: Instituto de Estudios para el Desarrollo y la Paz, Indepaz.

Herrera, L. M. (2006). El estado de la información sobre tenencia de la tierra para la formulación de politicas de tierra en América Latina. Organización de los Estados Americanos, Oficina de Desarrollo Sostenible y Medio Ambiente.

Jaramillo, S. (2009). Hacia una teoría de la renta del suelo urbano. 2. ${ }^{\text {e }}$ ed. Bogotá: Universidad de los Andes.

Lozano, R. (2007). Factores que inciden en el precio de las tierras de uso agrícola. Coastal Areas and Land Administration - Building the Capacity. 6th FIG Regional Conference. San José, Costa Rica 12-15. November 2007, 1-17.

Maldonado, M. (2006). Instrumentos de gestión del suelo. Algunos elementos del contexto. Notas de clase Instrumentos de Gestión del Suelo. Bogotá: Universidad de los Andes y Universidad Nacional de Colombia.

Mancero, B. G. (2012). Adquisición de empresas en el sector minero y petrolero colombiano. Colección de estudios en derecho minero y energético \#4. Departamento de Derecho Minero Energético. Boogotá: Universidad Externado de Colombia.

Páez, R. A. (2010). Bosques nacionales. Soporte de diversidad biológica y cultural. Informe preventivo. Bogotá: Instituto de Estudios del Ministerio Público y Procuraduría Delegada para Asuntos Ambientales y Agrarios. Colección de Asuntos Ambientales N. ${ }^{\circ} 9$. Procuraduría General de la República de Colombia.

Revéiz, E. (2013). El Estado estratega para el ordenamiento territorial. Bogotá: Academia Colombiana de Ciencias Económicas.

Santos, M. (1999). El territorio: un agregado de espacios banales. Pandero M. y Cebrián F. (Coords.): América Latina: lógicas locales, lógicas globales (p. 31- 39). Cuenca: Ed. Universidad de Castilla La Mancha. Colección Estudios.

Sassen, S. (2007). El reposicionamiento de las ciudades y regiones urbanas en una economía global: ampliando las opciones de políticas y gobernanza. Instituto de Estudios Urbano Regionales. Revista Latinoamérica de Estudios Urbano Regionales, 33(100), pp. 9-34. Pontificia Universidad Católica de Chile.

Sciara Á., Raposo I., Cafarell S. y Brun C. (2004). Infraestructuras y desarrollo regional. Novenas Jornadas "Investigaciones en la Facultad" de Ciencias Económicas y Estadística, noviembre 2004. pp. 1-19.

Silva, H. J. y Gonzales, S. D. N. (2010). El SIG en el corazón de Colombia. En: IGAC-ICDE, Sistema de Información Geográfica (pp. 89-97). Bogotá: Imprenta Nacional. 
Recepción: 5 de diciembre de 2015

Evaluación: 29 de marzo de 2016

Aprobación: 29 de abril de 2016

\begin{tabular}{l|l}
\hline 150 & Jahir A. Gutiérrez O., Gleidy A. Urrego E., Jefferson Carmona M.
\end{tabular} 